\title{
Low-complexity indoor UWB localization scheme using particle swarm optimization
}

\author{
${\text { Eiji } \text { Okamoto }^{1 a)} \text {, Manato Horiba }}^{1}$, Keita Nakashima ${ }^{1}$, \\ Toshiko Shinohara ${ }^{2}$, and Katsuhiko Matsumura ${ }^{2}$ \\ ${ }^{1}$ Department of Computer Science and Engineering, Graduate School of \\ Engineering, Nagoya Institute of Technology \\ Gokiso-cho, Showa-ku, Nagoya-shi 466-8555, Japan \\ ${ }^{2}$ Daifuku Co., Ltd. \\ 1225 Nakazaiji, Hino-cho, Gamo-gun, Shiga 529-1692, Japan \\ a) okamoto@nitech.ac.jp
}

Received August 28, 2014; Revised November 15, 2014; Published April 1, 2015

\begin{abstract}
We propose a low-complexity indoor localization scheme using a hybirid of particle swarm optimization (PSO) and Newton-Raphson (NR) search. The signal of global positioning system (GPS) can be utilized outdoors only, and other schemes are needed for indoor localization. A triangulation-based location estimation using ultra-wide band (UWB) signals between more than three reference terminals and the target node is widely used for centimeter-order localization. In particular, a time of arrival (TOA)-based least square (LS) estimation is popular because the balanced performance in terms of calculation complexity and the accuracy is obtained. However, when the height of reference terminals and the target node is close, the three-dimensional LS-based estimation tends to fall into a local-minimum solution and it needs an accurate initial value of search to keep the estimation performance, resulting in the calculation complexity increase. Therefore, in this paper, we adopt a particle swarm optimization (PSO) method which effectively searches in wide-area space and propose an LS-based localization scheme using the combination of PSO and NR method achieving lower calculation complexity. The improved performances are shown with comparing to conventional search schemes by computer simulations.
\end{abstract}

Key Words: location-based service, ultra-wide band, least square-based localization, particle swarm optimization, Newton-Raphson method

\section{Introduction}

Outdoor location-based services (LBS) such as map or navigation system in smartphone are widely used these days. The location information of those communication terminals is given by global positioning system (GPS) and various new service architectures based on the location information are being developed. One of the hot topics of LBS is a self-driving car system. To achieve the self-driving car an accurate location information is indispensable, otherwise car accidents may easily 
happen. The utilization of indoor location information is also expected to be developed. There are lots of new indoor services utilizing LBS. For example, a high-resolution location information enables segmented air condition, audio, and lighting supplies which contribute energy-saving and personalized environments. In long-distance wireless power transmission system, an accurate threedimensional location of the terminal to be supplied must be required. However, because the radio wave of GPS cannot be received in indoor, other measurement schemes are needed. As a popular one, ultra-wide band (UWB) radio wave-based triangulation between the target node whose location is unknown and the reference anchor nodes is utilized for centimeter-order localization. As the distance measurement between the target node and the anchor node, there are a few schemes such as time of arrival (TOA), time difference of arrival (TDOA), angle of arrival (AOA), and received signal strength (RSS). Among those schemes, TOA has a high measurement resolution and is often used [1]. Using more than three measured distance data the location of target node is estimated, and there are several estimation algorithms from the distance data to the location. The simplest one is the linear least square (LLS) scheme [2] which needs the least calculation complexity. However, for more accurate estimation, it is popular to use the maximum likelihood (ML) scheme or nonlinear least square (NLS or simply LS) scheme [3]. ML scheme provides the most exact location estimation with the requirements of higher calculation complexity and channel parameters. Meanwhile, LS scheme needs less calculation complexity and doesn't need the channel parameters. Hence, the LS scheme is widely used in indoor localization. Thus, in this study we focus on the LS-based localization scheme. There are lots of optimization algorithms applicable for LS-based localization and among them, the full grid search, Newton-Raphson (NR) algorithm, and a nonlinear optimization are often adopted. We have considered a hybrid use of NR and grid search in which a rough grid search is conducted in advance and its result is used as the initial value of NR fine search in the second stage [4]. This scheme can balance the calculation complexity and the accuracy. However, to get the result of NR search converged a relatively exact initial value has to be supplied. In general, the three-dimensional localization tends to need higher complexity [5]. In particular, when the height of the target and anchor nodes is close in three-dimensional localization, more exact initial value is needed to avoid making a local optimal solution output by NR scheme, resulting in the calculation complexity increase in grid search. This happens in the low-height anchor node case which is rather general because the roof of rooms is usually not so high. In addition to that, the indoor terminals whose location is to be estimated moves in many cases such as a mobile phone with person or a forklift in industrial plant. Hence, to achieve the LBS, a short time delay estimation with lower calculation complexity is needed.

To solve this problem, in this paper we adopt a particle swarm optimization (PSO) algorithm [6] and propose a lower-complexity LS-based localization scheme with the hybrid of PSO and NR optimization. PSO is one of the metaheuristic ( $\mathrm{MH})$ optimization schemes [7] and a wide-range optimization is efficiently conducted. Other than PSO, there are several MH optimization methods such as genetic algorithm (GA) [8], simulated annealing (SA) [9], and tabu search (TS) [10]. Among these methods, PSO is adopted in this study because PSO is suitable for a wide-area search on continuous variables (three-dimensional coordinate) with lower complexity. As the conventional studies, the PSO localization with RSS signals has been proposed in $[11,12]$. However, the estimation error is relatively high because RSS signals are utilized. The TDOA-based exact localization using PSO has been studied in $[13,14]$ and the ML estimation using PSO has been studied in [15]. However, those studies are two-dimensional localization and the height problem is not considered. We also have proposed the PSO and NR optimization scheme in [16]. However, the performance was degraded at the lower and higher heights of the target node because the estimation accuracy of height direction was lowered, and the effect was limited. In the proposed scheme here, the first estimation results are obtained by the PSO scheme with low calculation complexity, and then, the result is handled to the NR fine search with some modification and the fine search is conducted. By this algorithm, the complexity reduction and the accurate estimation is simultaneously achieved.

In the following, the system model and LS estimation is introduced in Section 2, the conventional and the proposed optimization schemes for LS estimation are described in Section 3, and the numerical results are illustrated in Section 4. Finally, the conclusions are drawn in Section 5. 


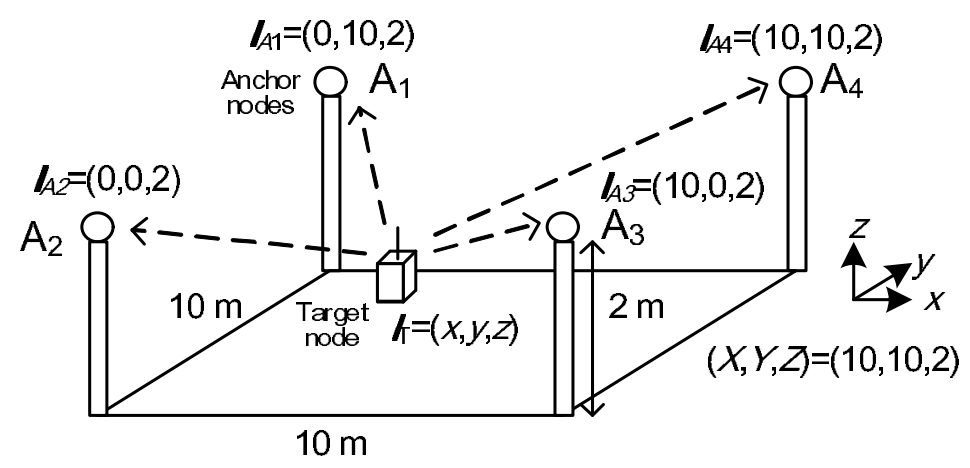

Fig. 1. Three-dimensional sensor field in this study.

\section{System model and least square localization}

Figure 1 shows the indoor location estimation system in this study. The three dimensional sensor field with $X=Y=10, Z=2$ meter is considered and the four anchor nodes whose positions are known are located at the four corners with the height of $Z=2$. The location of $i$-th anchor node is denoted by $\mathbf{l}_{A_{i}}=\left(x_{i}, y_{i}, z_{i}\right), 1 \leq i \leq N$ where $N=4$ is the number of anchor nodes. One target node whose location is to be estimated exists and it broadcasts an ultra-wide band (UWB) beacon. Four anchor nodes receive it and calculate the distance between each anchor node and the target node in TOA manner, and a central unit estimates the target node location by triangulation using those distance data. In this paper, we focus on the construction of position estimation scheme, and the UWB beacon transmission model is assumed to be the same as [17] scheme. In [17], based on Howard's measurement scheme [18], a chirp-waveform UWB pulse is transmitted and a coherent detection using network analyzer is adopted, and this transmission model is assumed in this study. The antennas of each node are assumed as an isotropic antenna.

The true location of the target node is denoted by $\mathbf{l}_{T}=(x, y, z)$. Then, the true distance between the anchor node $A_{i}$ and the target node $T$ is denoted by

$$
d_{i}=\left\|\mathbf{l}_{T}-\mathbf{l}_{A_{i}}\right\|=\sqrt{\left(x-x_{i}\right)^{2}+\left(y-y_{i}\right)^{2}+\left(z-z_{i}\right)^{2}}
$$

When it is measured at $A_{i}$, the error is added and it becomes $\hat{d}_{i}=d_{i}+\varepsilon_{i}$ where $\varepsilon_{i}$ is the noise component in measurements, caused by the multipath reception of UWB beacon (Eq. (2) in [17]). It is assumed in this study that the propagation channel between all anchor nodes and the target is in line-of-sight (LOS) environments. According to [17] and [19], in this UWB measurement $\hat{d}_{i}$ has a Gaussian distribution with the error mean of $m_{i}=m_{\mathrm{LOS}} \log \left(1+d_{i}\right)$ and the variance $\sigma_{i}^{2}$. Here, $m_{\mathrm{LOS}}$ and $\sigma_{i}^{2}$ are the parameters depending on the bandwidth of UWB signal. The probability density function (pdf) of $\hat{d}_{i}$ is given by

$$
p\left(\hat{d}_{i} \mid d_{i}\right)=\frac{1}{\sqrt{2 \pi} \sigma_{i}} \exp \left[-\frac{\left(\hat{d}_{i}-d_{i}-m_{i}\right)^{2}}{2 \sigma_{i}^{2}}\right]
$$

Therefore, the accuracy of TOA distance can be raised by the multiple signal measurements at each anchor node. It is assumed that the target node does not move during one position estimation. Then, when the anchor node $A_{i}$ measures the distance $M$ times and $k$-th distance is denoted by $\hat{d}_{i, k}$, the measurement results of distance $\hat{d}_{i}$ is given by

$$
\hat{d}_{i}=\frac{1}{M} \sum_{k=1}^{M} \hat{d}_{i, k}
$$

which is more reliable than a single measurement. For exploiting the $M$-time measurements more, the integration and averaging of the UWB waveforms will be effective. However, because it needs more memory and signal processing, the averaging of each measured distance of (3) is adopted in this study. Using $\hat{d}_{i}$, the estimated location of the target node is calculated in the LS manner by 


$$
\hat{\mathbf{l}}_{T}=\underset{x, y, z}{\arg \min } \sum_{i=1}^{N}\left[\sqrt{\left(x-x_{i}\right)^{2}+\left(y-y_{i}\right)^{2}+\left(z-z_{i}\right)^{2}}-\hat{d}_{i}\right]
$$

This equation is a nonlinear optimization problem and there are several solution algorithms. Some of typical schemes and the proposed scheme are introduced in the next section.

\section{Optimization schemes in LS-based localization}

The LS estimation of (4) needs less calculation complexity than that of ML estimation. Different from ML scheme, the LS scheme doesn't need channel parameters in calculation but can obtain relatively good estimation performance. However, it still needs the nonlinear solution search as well as ML scheme. As the conventional algorithm, grid search and the sequential search of gird and NR schemes are introduced here, and the proposed scheme further reducing the calculation complexity is also described.

\subsection{Grid search}

Grid search is the simplest solution search algorithm. In the grid search, the solution is fully searched at the discrete and equally-spaced grid in the search space in general. For (4), the grid search becomes

$$
\begin{array}{r}
\hat{\mathbf{l}}_{T}=\underset{j, k, l}{\arg \min } \sum_{i=1}^{N}\left[\sqrt{\left(j \delta d-x_{i}\right)^{2}+\left(k \delta d-y_{i}\right)^{2}+\left(l \delta d-z_{i}\right)^{2}}-\hat{d}_{i}\right] \\
0 \leq j \leq\lfloor X / \delta d\rfloor, 0 \leq k \leq\lfloor Y / \delta d\rfloor, 0 \leq l \leq\lfloor Z / \delta d\rfloor
\end{array}
$$

where $\delta d$ is the grid interval and $\lfloor x\rfloor$ means the maximum integer less than $x$. The search number of (5) becomes $(\lfloor X / \delta d\rfloor+1)(\lfloor Y / \delta d\rfloor+1)(\lfloor Z / \delta d\rfloor+1)$, and the estimation accuracy and the calculation complexity are increased when $\delta d$ is smaller.

\subsection{Seqeuntial search of grid and Newton-Raphson}

NR scheme is an iterative optimal solution search algorithm of equation and it needs an appropriate initial value close to the true solution value to avoid converging to local optimal values. Hence, in (4) calculation, the coarse search of Subsection 3.1 using a relatively large grid is conducted at first, and its result is used as the initial value of NR search. Then, the fine search is conducted by NR search in the second stage. When the object function of LS estimation by NR search is denoted as

$$
f(x, y, z)=\sum_{i=1}^{N}\left[\sqrt{\left(x-x_{i}\right)^{2}+\left(y-y_{i}\right)^{2}+\left(z-z_{i}\right)^{2}}-\hat{d}_{i}\right]
$$

then, the estimation result is obtained by

$$
\hat{\mathbf{l}}_{T}=\underset{x, y, z}{\arg \min } f(x, y, z) .
$$

When a neighborhood solution of $\hat{\mathbf{l}}_{T}$ is given as $\hat{\mathbf{l}}_{T}=(\hat{x}, \hat{y}, \hat{z})$, the update equation of NR scheme and the difference parameters are obtained as follows.

$$
\begin{gathered}
\hat{\mathbf{l}}_{T, \text { new }}=\hat{\mathbf{l}}_{T}+\delta \mathbf{l}, \delta \mathbf{l}=(\delta x, \delta y, \delta z) \\
{\left[\begin{array}{lll}
\frac{\partial g_{1}\left(\hat{\mathbf{l}}_{T}\right)}{\partial x} & \frac{\partial g_{1}\left(\hat{\mathbf{l}}_{T}\right)}{\partial y} & \frac{\partial g_{1}\left(\hat{\mathbf{l}}_{T}\right)}{\partial z} \\
\frac{\partial g_{2}\left(\hat{\mathbf{l}}_{T}\right)}{\partial x} & \frac{\partial g_{2}\left(\hat{\mathbf{l}}_{T}\right)}{\partial y} & \frac{\partial g_{2}\left(\hat{\mathbf{l}}_{T}\right)}{\partial z} \\
\frac{\partial g_{3}\left(\hat{\mathbf{l}}_{T}\right)}{\partial x} & \frac{\partial g_{3}\left(\hat{\mathbf{l}}_{T}\right)}{\partial y} & \frac{\partial g_{3}\left(\hat{\mathbf{l}}_{T}\right)}{\partial z}
\end{array}\right]\left[\begin{array}{l}
\delta x \\
\delta y \\
\delta z
\end{array}\right]=\left[\begin{array}{l}
-g_{1}\left(\hat{\mathbf{l}}_{T}\right) \\
-g_{2}\left(\hat{\mathbf{l}}_{T}\right) \\
-g_{3}\left(\hat{\mathbf{l}}_{T}\right)
\end{array}\right]} \\
\left\{\begin{array}{l}
g_{1}(x, y, z)=\frac{\partial f(x, y, z)}{\partial x}=0 \\
g_{2}(x, y, z)=\frac{\partial f(x, y, z)}{\partial y}=0 \\
g_{3}(x, y, z)=\frac{\partial f(x, y, z)}{\partial z}=0
\end{array}\right.
\end{gathered}
$$


Here, (9) is the derivative of (6) and the solution satisfying (9) is searched by (7) and (8). The iterative search is conducted using $\delta \mathbf{l}$ calculated by LU decomposition of (8), and the update of (7) and (8) is iterated from the initial value of coarse search results on (5) until

$$
f\left(\hat{\mathbf{l}}_{T, \text { new }}\right) \geq f\left(\hat{\mathbf{l}}_{T}\right)
$$

is satisfied. Finally, $\hat{\mathbf{l}}_{T}$ at that point is output as the solution. Note that the estimated coordinate is limited in the range of sensor field, that is, $x, y$, or $z$ is forced to be zero when it is negative and to be $X, Y$, or $Z$ when it is over that value.

\subsection{Particle swarm optimization-based search}

In the scheme of Subsection 3.2, when the heights of the target node $z$ becomes larger, the estimation result tends to converge to local optimal value that means the result having large estimation error (see Fig. 5). As a result, the small $\delta d$ of (5) was needed to generate an accurate initial value and the calculation complexity was increased. Hence, we consider applying MH search because it is efficient for wide-area search [7]. GA, SA, TS, and PSO are popular in MH and each method has different characteristics. In general, GA, SA, and TS are suitable for combination optimization and an increased number of states is needed when applied to continuous optimization. It is known that SA tends to need long time to converse the search results and TS needs less time. PSO can deal with continuous variable optimization and need less calculation complexity in general. Here, the optimization function of the localization on (6) has a continuous variables of three-dimensional coordinate, and the lowcomplexity estimation is needed to achieve a real-time estimation. Thus, we adopt PSO method in this study. The PSO-based LS estimation is described in the following.

The object function to be minimized in PSO search is the same as (6) and let $N_{p}$ as the number of particles. The location and velocity of the particle $i$ at time $n$ are defined, respectively, by

$$
\begin{aligned}
& \mathbf{l}^{i}(n)=\left[x^{i}(n), y^{i}(n), z^{i}(n)\right], 1 \leq i \leq N_{p} \\
& \mathbf{v}^{i}(n)=\left[v_{x}^{i}(n), v_{y}^{i}(n), v_{z}^{i}(n)\right]
\end{aligned}
$$

Then, the personal best of $i$-th particle is given by

$$
\mathbf{p}^{i}=\left(p_{x}^{i}, p_{y}^{i}, p_{z}^{i}\right), f\left(\mathbf{p}^{i}\right) \leq f\left(\mathbf{l}^{i}(n)\right), 0 \leq n \leq n_{c}
$$

where $n_{c}$ is the current time. The global best of all particles is given by

$$
\mathbf{g}=\left(g_{x}, g_{y}, g_{z}\right), f(\mathbf{g}) \leq f\left(\mathbf{p}^{i}\right), 0 \leq i \leq N_{p}
$$

and the local best $\mathbf{b}^{i}=\left(b_{x}^{i}, b_{y}^{i}, b_{z}^{i}\right)$ is configured as a ring connection for $i$-th particle such as

$$
\begin{aligned}
& f\left(\mathbf{b}^{i}\right) \leq f\left(\mathbf{p}^{j}\right), 0 \leq i \leq N_{p}, \\
& \left(i-1+N_{p}\right) \bmod N_{p} \leq j \leq\left(i+1+N_{p}\right) \bmod N_{p}
\end{aligned}
$$

Then, the update of $i$-th particle in the $x$ axis is given by

$$
\left\{\begin{array}{l}
v_{x}^{i}(n+1)=w v_{x}^{i}(n)+\rho_{1}\left\{p_{x}^{i}-x^{i}(n)\right\}+\rho_{2}\left\{b_{x}^{i}-x^{i}(n)\right\} \\
x^{i}(n+1)=x^{i}(n)+v_{x}^{i}(n)
\end{array}\right.
$$

where $w, \rho_{1}$, and $\rho_{2}$ are the behavior control parameters of particle. The location and velocities of other axes $v_{y}^{i}, v_{z}^{i}, y^{i}$, and $z^{i}$ are calculated in the same way. In this study, by the heuristic adjustment for the sensor field of Fig. 1, these parameters are configured as follows: $w$ of (15) is set as 0.5, $\rho_{1}$ and $\rho_{2}$ are set as $\rho_{1}, \rho_{2} \in \operatorname{RND}[0,0.42]$ for $x$ and $y$ axes, and as

$$
\rho_{1} \in \operatorname{RND}[0,0.42], \rho_{2} \in \operatorname{RND}\left[0, \frac{0.42 Z}{X}\right]
$$

for $z$ axis because $z$ space is smaller than $x$ and $y$ spaces. Here, $\operatorname{RND}[a, b]$ denotes the uniform random number in the range of $[a, b]$. The initial value of $i$-th location and velocity is randomly given by 


$$
\begin{aligned}
& \mathbf{l}^{i}(0)=(\operatorname{RND}[0, \mathrm{X}], \operatorname{RND}[0, \mathrm{Y}], \operatorname{RND}[0, \mathrm{Z}]) \\
& v_{x}^{i}(0), v_{y}^{i}(0), v_{z}^{i}(0) \in \operatorname{RND}[0,0.4]-0.2
\end{aligned}
$$

The global best solution $\mathbf{g}$ is the estimated output at time $n$. The terminating condition is set as $f(\mathrm{~g}) \leq 10^{-4}$ which is close to the exact solution and the maximum iteration number is set as $n_{\max }$. In this termination condition, because the iteration reaches $n_{\max }$ in almost all cases, the calculation complexity and the accuracy can be controlled by $n_{\max }$. Here, as with Subsection 3.2, the estimated coordinate $g_{x}, g_{y}$, or $g_{z}$ is modified to the boundary value of the sensor field if it is over that range.

\subsection{Proposed search scheme}

To reduce the calculation complexity while sustaining the estimation accuracy, we propose a sequential search scheme in which the result of rough PSO search is used in NR scheme. In the proposed scheme, the global best solution $\mathbf{g}$ of PSO with relatively small $n_{\max }$ is passed to NR scheme as the initial value and the sequential LS estimation is conducted. Because the accuracy of PSO search is higher than that of grid search at the same calculation complexity in general, the better estimation performance is expected. However, we found the problem that the estimation error became relatively large in $z$ direction when the result of PSO search was directly handled to NR search. Then, by numerical analysis, it is found that when the initial $\hat{z}$ value of NR search is larger than the actual $z$ value of target node, the estimation error increases in $z$ direction. Therefore, in the proposed scheme, the scaled-down $z$ value of PSO search is handled to the initial value of NR fine search as follows:

$$
\hat{z}=0.3 g_{z}
$$

which is derived by numerical analysis. The derivation of this scale down parameter is discussed with numerical analysis in the next section.

\section{Numerical results}

The performances of calculation complexity and estimation accuracy of the proposed scheme are evaluated by computer simulations. The UWB measurement environment is assumed as the same as [17], where a chirp-waveform UWB pulse is transmitted and a complex value of received signal is stored. In practice, the characteristics of $\hat{d}_{i, k}$ in (3) will be changed according to the UWB system such as performance of analog to digital (A/D) converter, antenna pattern, and UWB waveform. The three-dimensional root mean square error (RMSE) is calculated as the estimation error. Table I shows the simulation conditions. The target node at the location of $(x, y, z)$ sends beacons 30 times $(M=30)$ and the anchor nodes receive them. The RMSE is calculated after 1000 times trial at each target node location. The target node location is changed at every $0.5 \mathrm{~m}$ for $x$ and $y$ axes while $z=1$ $\mathrm{m}$ is fixed, and RMSE is calculated on all $x-y$ plane. The UWB signal bandwidth is assumed as 3 $\mathrm{GHz}$ and the channel parameter is configured by the experimental results of [17]. This $3 \mathrm{GHz} \mathrm{UWB}$ pulse is transmitted and received in LOS environments in the sensor field in Fig. 1. As the calculation complexity, the calculation of (6) is defined as one search, and the average number of calculation on one location estimation is compared.

Table I. Simulation conditions.

\begin{tabular}{|c|c|}
\hline Sensor field & $X=Y=10 \mathrm{~m}, Z=2 \mathrm{~m}$ \\
\hline Number of anchor nodes & $N=4$ (Fig. 1$)$ \\
\hline Target node location & $\begin{array}{l}0 \leq x \leq X \text { and } 0 \leq y \leq Y \\
\text { on every } 0.5 \mathrm{~m} \text { grid, } z=1 \mathrm{~m}\end{array}$ \\
\hline Number of TOA measurements per one estimation & $M=30$ \\
\hline Number of simulation for RMSE calculation & 1,000 times \\
\hline UWB bandwidth & $3 \mathrm{GHz}$ \\
\hline UWB channel parameter & $m_{\mathrm{LOS}}=4.0 \times 10^{-3}, \sigma_{i}^{2}=4.5 \times 10^{-2}[17]$ \\
\hline Channel model & Line of sight $(\mathrm{LOS})$ \\
\hline Number of particles & $N_{p}=10$ \\
\hline
\end{tabular}




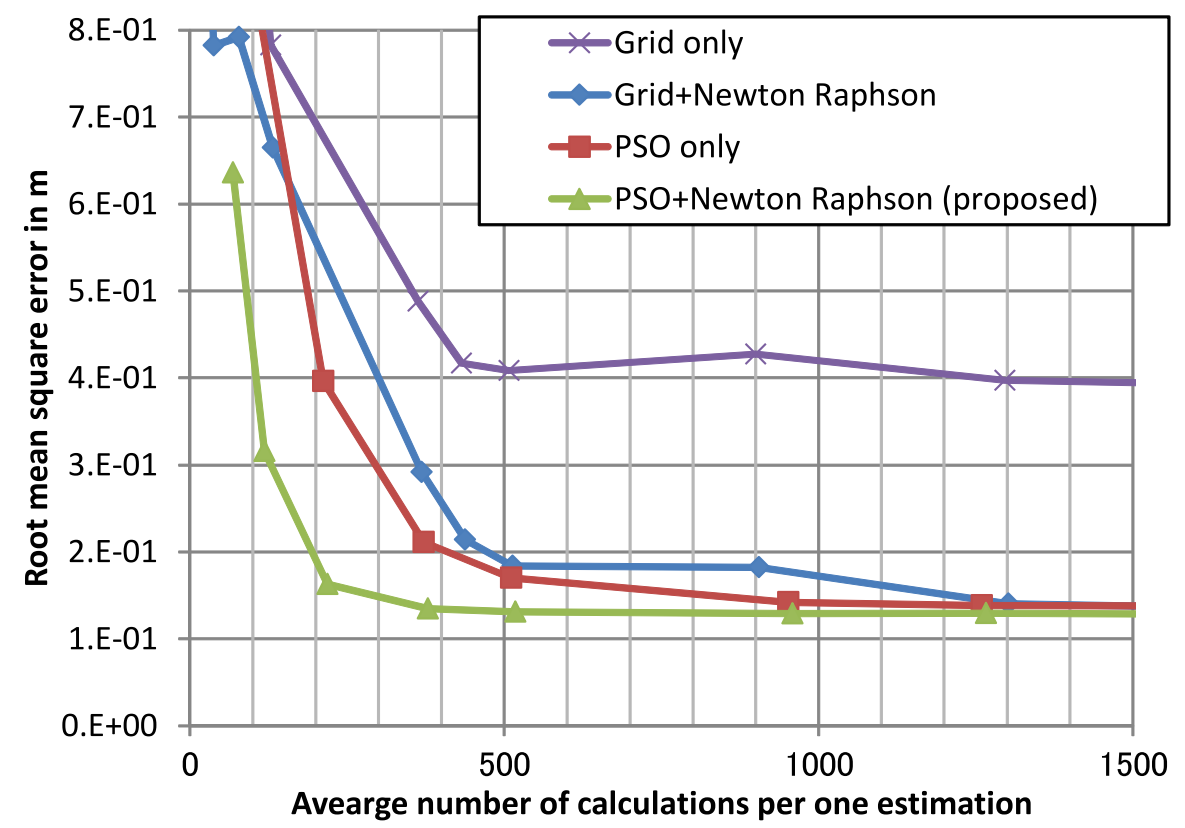

Fig. 2. RMSE performance comparison of the proposed and conventional schemes versus calculation complexity.

Table II. Relationship between parameter settings and required calculation complexity.

\begin{tabular}{|c|c|c||c|c|c|}
\hline & \multicolumn{2}{|c||}{ average number of calculation } & & \multicolumn{2}{c|}{ average number of calculation } \\
\hline$\delta d \mathrm{~m}$ & grid & grid+NR & $n_{\max }$ & PSO & proposed PSO+NR \\
\hline \hline 8.0 & 4.0 & 9.6 & & & \\
\hline 3.0 & 32.0 & 37.6 & & & \\
\hline 2.0 & 72.0 & 77.3 & 5 & 62.0 & 68.1 \\
\hline 1.5 & 128.0 & 131.6 & 10 & 112.0 & 118.4 \\
\hline 1.0 & 363.0 & 368.6 & 20 & 212.0 & 218.6 \\
\hline 0.9 & 432.0 & 437.4 & 36 & 371.9 & 378.6 \\
\hline 0.8 & 507.0 & 512.5 & 50 & 511.6 & 518.2 \\
\hline 0.7 & 900.0 & 905.0 & 100 & 1004.7 & 1011.4 \\
\hline 0.6 & 1296.0 & 1302.0 & 150 & 1487.9 & 1495.3 \\
\hline
\end{tabular}

The average RMSE of $x-y$ plane versus average calculation complexity is shown in Fig. 2 where the grid search in Subsection 3.1, the sequential scheme of grid and NR in Subsection 3.2, and PSO scheme are compared as the conventional schemes. Here, the relationship between the average number of calculation and the grid width $\delta d$ or the maximum number of iteration $n_{\max }$ in PSO is listed in Table II. The result of Fig. 2 shows that the RMSE performances are improved according to the calculation increase in all schemes. This is because the calculated location approaches to the true location with a small grid $\delta d$ in the grid-based search, and the each particle become easier to converge into the true location with the increased $n_{\max }$ in PSO scheme. When compared with other schemes, the grid scheme has the worst performance and it needs further calculation complexity with much smaller $\delta d$ for improvement. The hybrid scheme of grid and NR has better performance than the grid scheme because of the fine search of NR scheme. However, the RMSE is increased when the accuracy of initial value is not good. On the other hand, compared to the grid and NR scheme, PSO-based schemes have relatively better performance at the lower complexity that means the effective particle initial search is valid in the LS-based localization. Among all schemes, the proposed PSO and NR scheme needs less calculation complexity to obtain the same RMSE. It can be seen that over 1300 calculation the RMSE of the proposed scheme becomes almost the same as the hybrid scheme of grid and NR, and that the proposed scheme has better performance below that calculation complexity. 


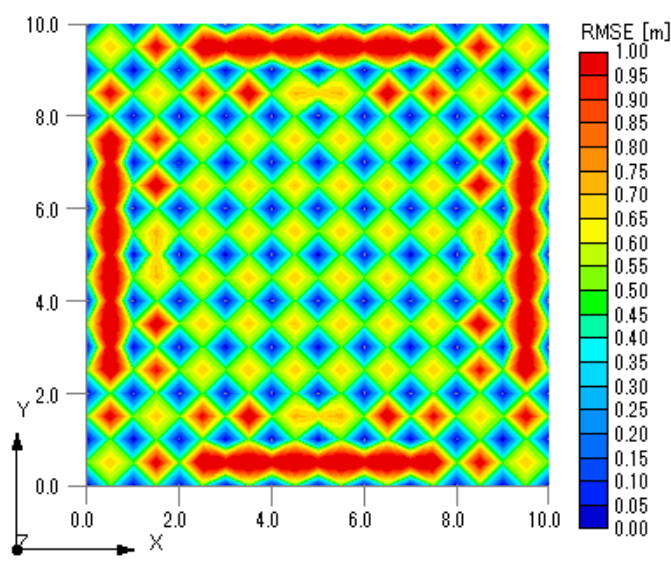

(a)

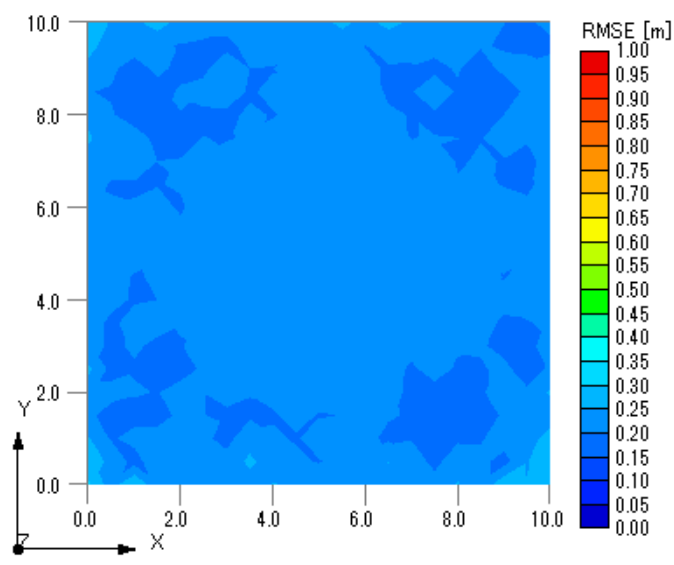

(c)

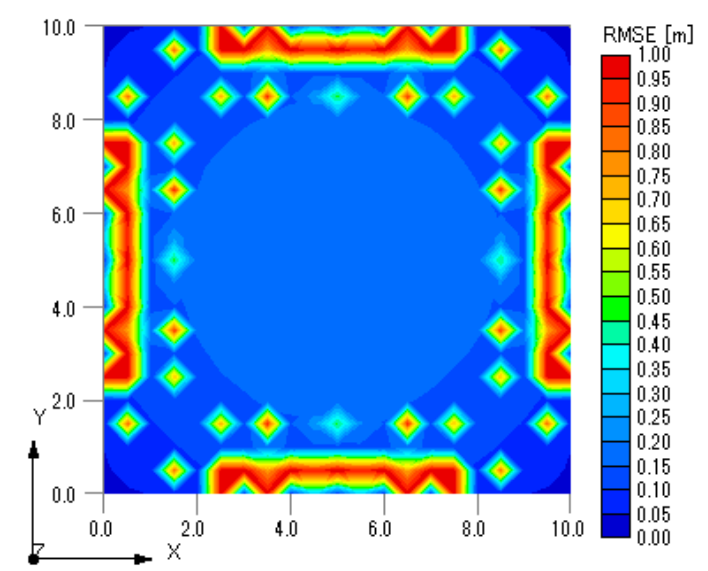

(b)

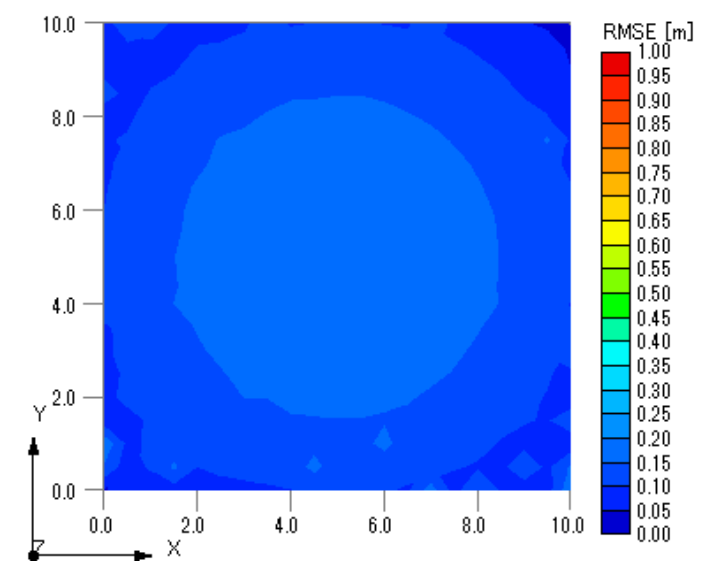

(d)

Fig. 3. RMSE performances on $x-y$ plane at calculation complexity of 370; (a) grid search, (b) hybrid search of grid and NR, (c) PSO scheme, (d) proposed hybrid search of PSO and NR.

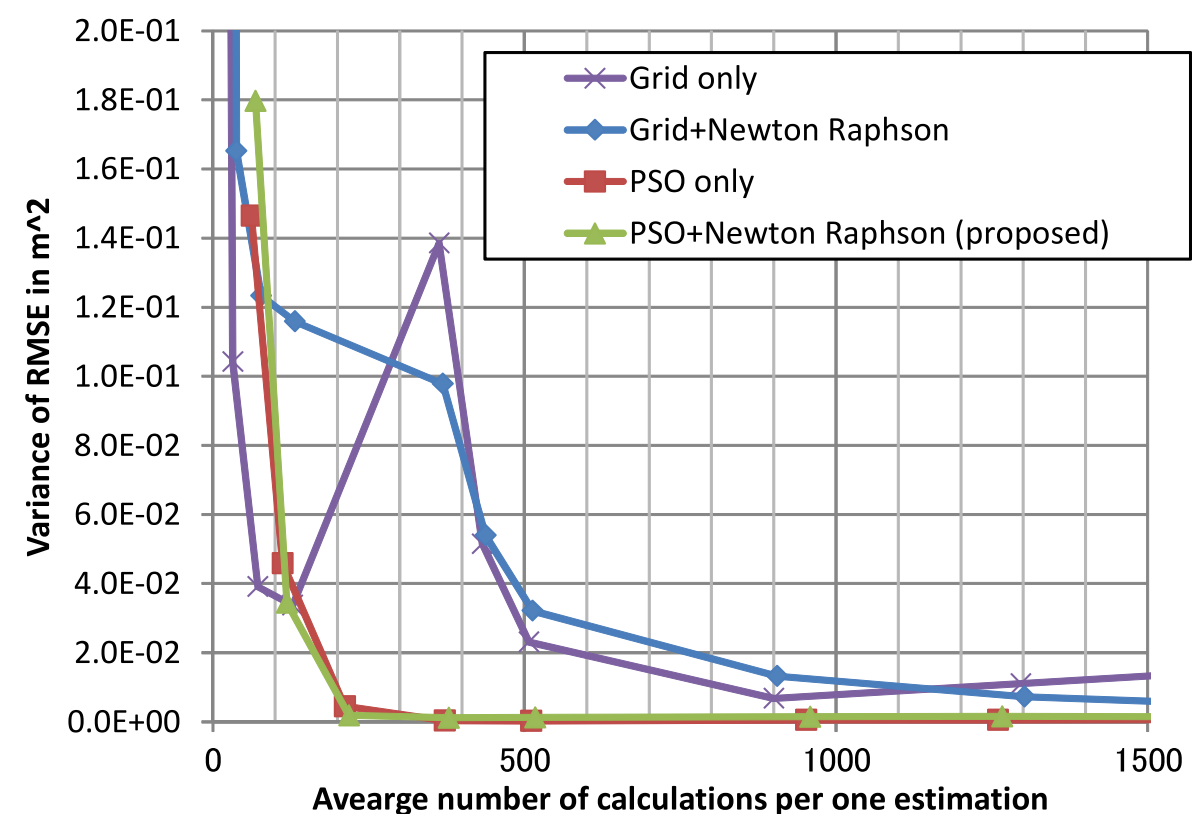

Fig. 4. Performance comparison of RMSE variance on $x-y$ plane in the proposed and conventional schemes versus calculation complexity. 


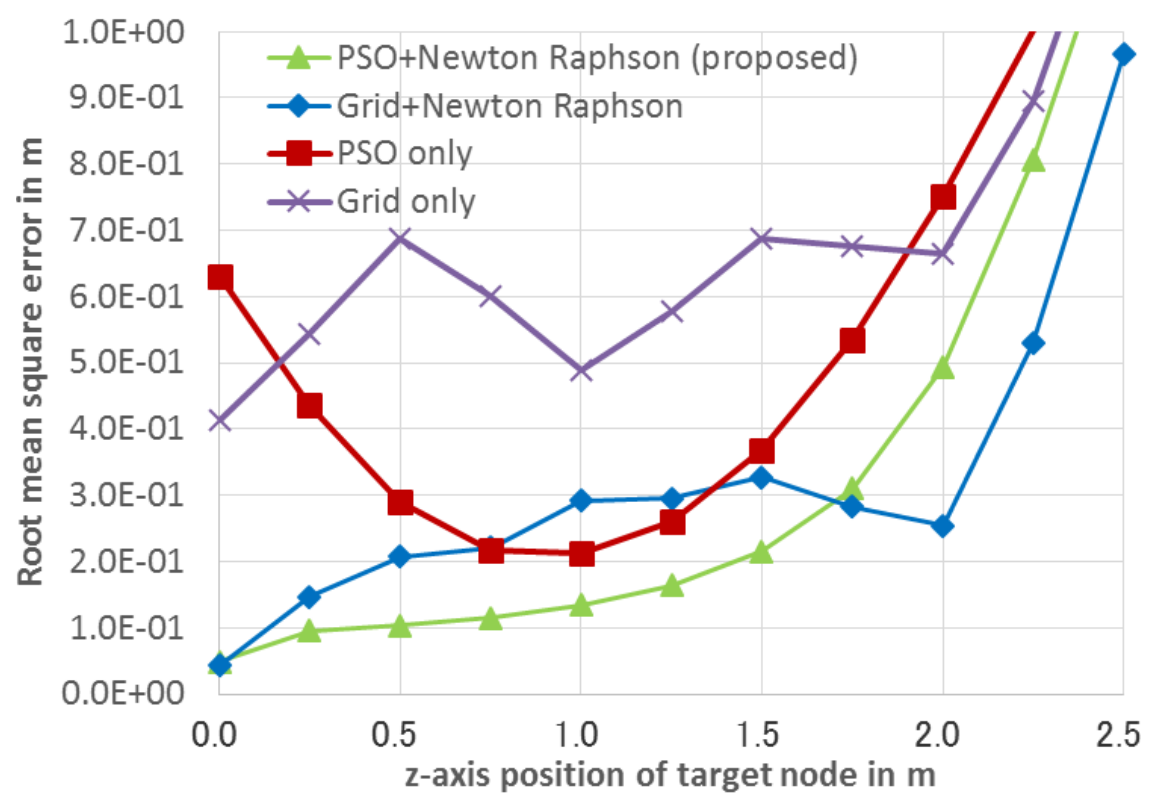

Fig. 5. RMSE performance comparison at calculation complexity of 370 versus the height of target node.

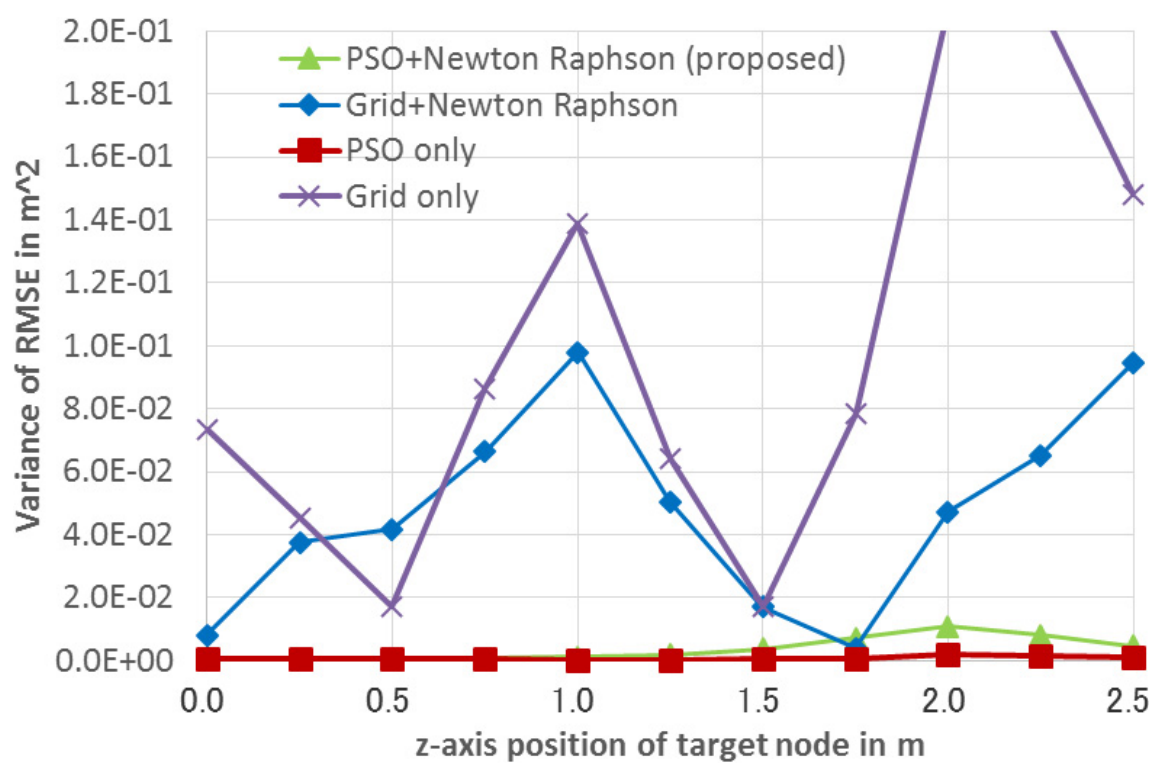

Fig. 6. Performance comparison of RMSE variance at calculation complexity of 370 versus the height of target node.

In addition, the proposed scheme has the best RMSE performance of four schemes and the RMSE is converged to lower bound with the calculation complexity of $370,1 / 3$ times of the hybrid scheme of grid and NR. This means the PSO scheme effectively produces a good initial value to NR scheme with less complexity, and the proposed combination of PSO initial search and NR fine search is effective. Hence, the proposed scheme achieves an accurate LS estimation with lower complexity. Note that in the configuration of Fig. 1 where all anchor nodes have the same height, the three-dimensional LLS estimation [2] generates an extreme coordinate value (in our simulation $(10,10,0)$ with the average RMSE of $7.77 \mathrm{~m}$ ), and neither single LLS scheme nor LLS with NR scheme work correctly.

Next, the RMSE performances of four schemes on $x-y$ plane at $\delta d=1.0$ or $n_{\max }=36$ whose calculation complexity becomes around 370 as shown in Table II are compared. The results of Fig. 3 show that there are irregular and relatively large errors in Fig. 3(a) due to the grid-based search. In Fig. 3(b), the center area is improved thanks to NR fine search but there still exists a large error in the side area because the initial value is not sufficiently accurate. On the other hand, as shown 


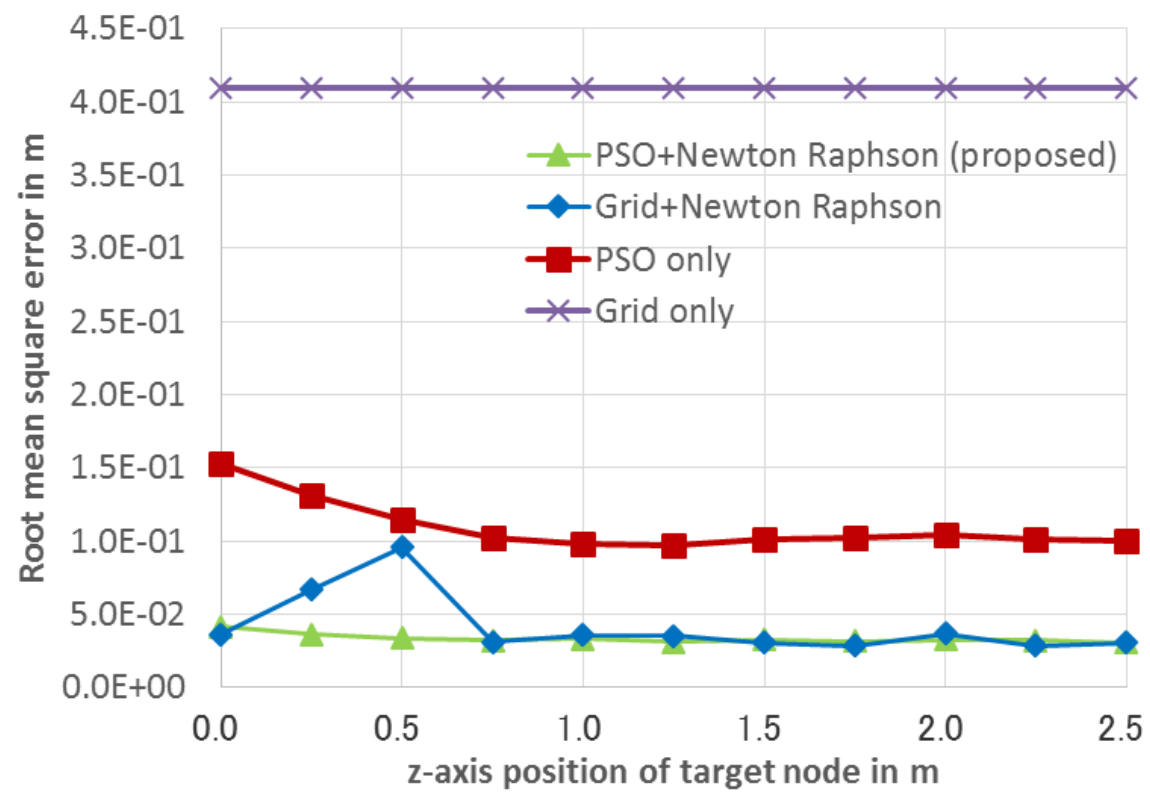

Fig. 7. Two-demensional (x-y) RMSE performance at calculation complexity of 370 versus the height of target node.

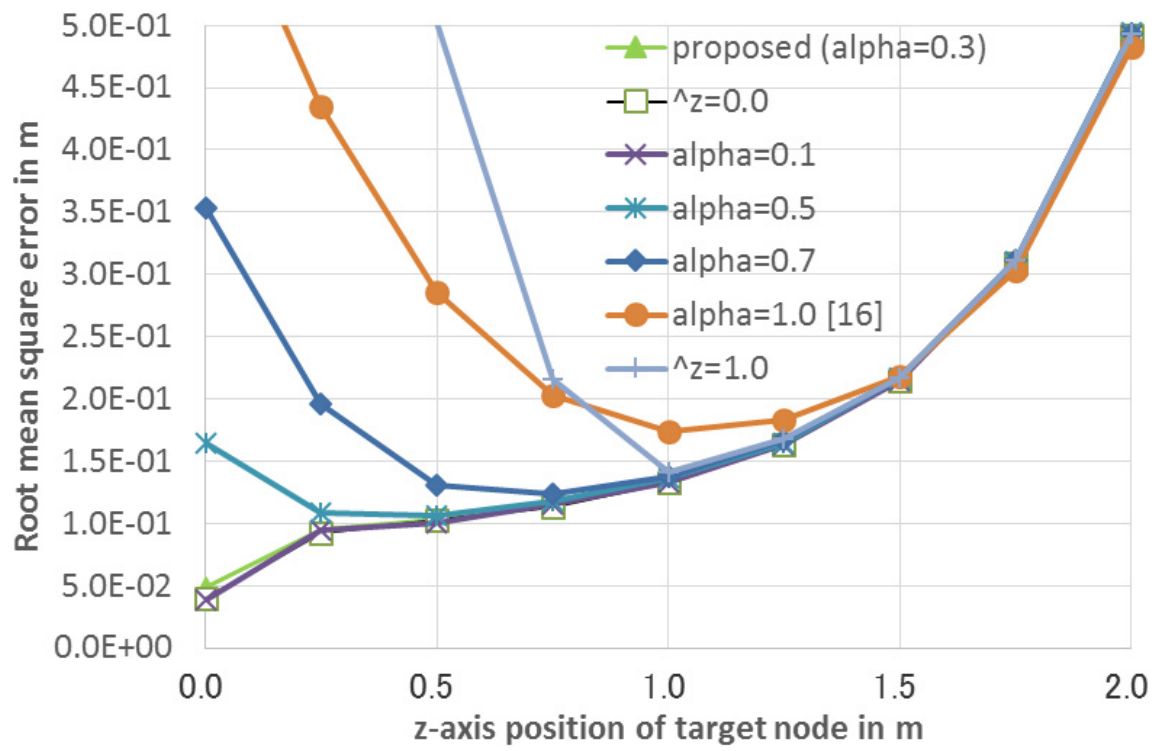

Fig. 8. RMSE performance comparison versus the height of target node with the parameter of initial value of $\hat{z}$.

in Figs. 3(c) and (d), PSO schemes have averagely good RMSE performances in all areas and the proposed scheme has the best performance.

To confirm the uniform performance of the proposed scheme, the variance of RMSE on $x-y$ plane of Fig. 3 and of the simulation in Fig. 2 is calculated. When the variance is small, it means the RMSE is more uniform regardless of the $x-y$ position of the target node. As shown in Fig. 4 , in the grid search the variance largely changes because the search point depends on $\delta d$ and the results become irregular as shown in Fig. 3(a). In the conventional grid and NR search, the variance is still large due to the initial discrete grid search. On the other hand, PSO searches have relatively small variance because the effective wide-area search can be conducted by particle search, and as a result, almost the uniform estimation results are obtained after more than 200 calculation in PSO and the proposed schemes. Around 100 calculation, the variance performance of the proposed scheme is worse than the PSO scheme because the PSO estimation is stably degraded, while some estimation is degraded in the proposed scheme, as shown in Fig. 3. Thus, the proposed scheme has averaged and good RMSE 


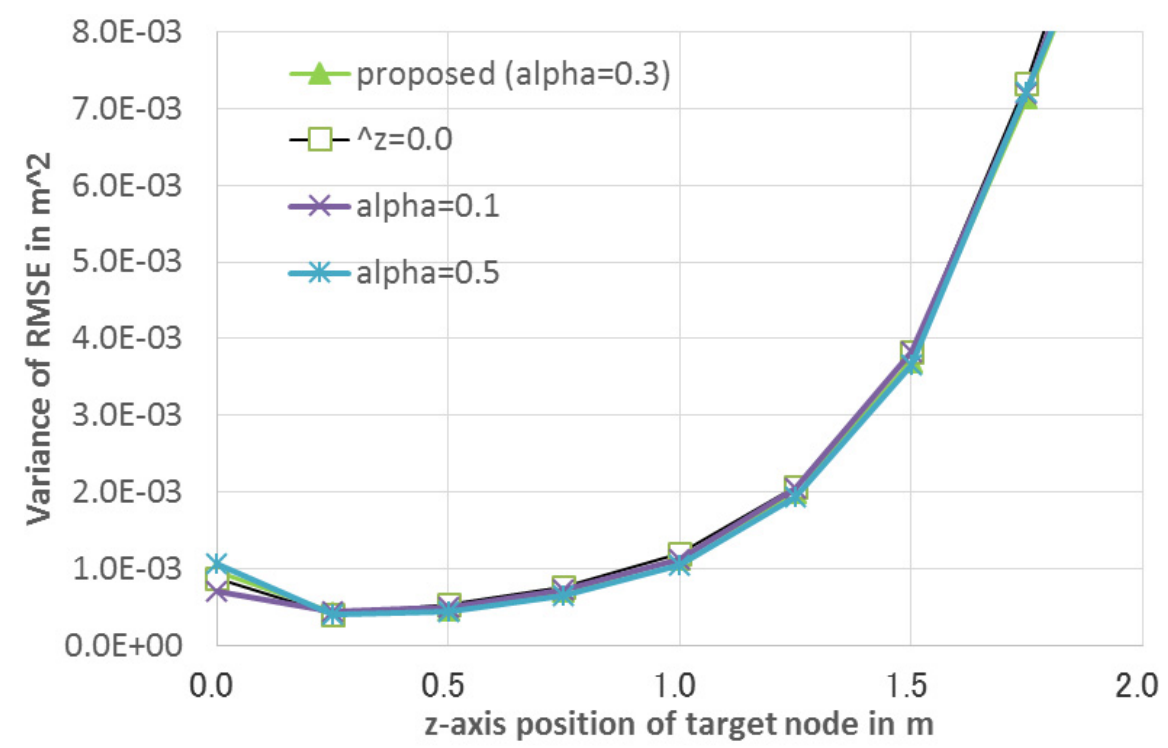

(a)

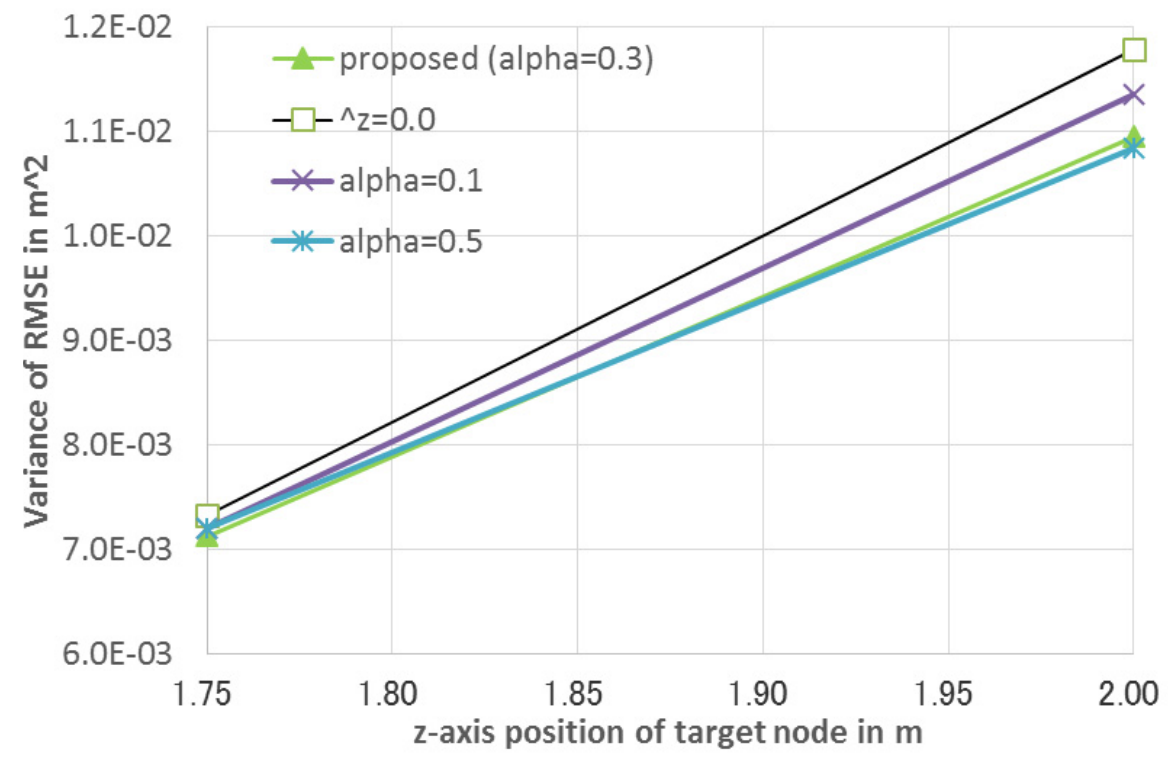

(b)

Fig. 9. Performance comparison of RMSE variance versus the height of target node with the parameter of initial value of $\hat{z}$; (a) $0 \leq z \leq 1.75$, (b) $1.75 \leq z \leq 2$.

performance.

Then, the average RMSE on $x-y$ plane versus the $z$ height $(0 \leq z \leq 2.5)$ of the target node when the calculation complexity is fixed at around 370 is calculated. The simulation conditions are the same as Table I except the $z$ height. Note that $n_{\max }$ is reduced to 35 in the proposed scheme to reduce the complexity to 368 , and that the RMSE until the height of $2.5 \mathrm{~m}$ is shown to view the performance trends for $z$. Here, because the estimated $z$ is limited to $Z$, the RMSE of all schemes is degraded over the height of $2.0 \mathrm{~m}$. The results of Fig. 5 shows that the RMSE of the proposed scheme is similar or less than that of the conventional grid and NR scheme. In particular, it can be seen that RMSE at the height between 0.5 and $1.5 \mathrm{~m}$, which is a practical height of the target node in indoor environments like the configuration of Table I, is a half. As well as Fig. 4, the RMSE variance versus $z$ height is calculated as shown in Fig. 6. The variance of the conventional grid-based schemes change largely for $z$ value because the initial grid search is discrete, while that of the proposed scheme is much smaller below $z=1.5 \mathrm{~m}$ and the proposed scheme has better performance except $1.75 \mathrm{~m}$ of $z$. Therefore, it is clarified that the proposed scheme achieves the low-complexity, accurate, and stable LS estimation compared to the conventional schemes except higher $z$ area close to $Z$ of anchor nodes. 
Here, to investigate the performance degradation at the roof area, we calculated the two-dimensional $x-y$ RMSE in the same simulation. As shown in Fig. 7, it is seen that the RMSE of the proposed scheme is the same as or less than that of the conventional grid and NR scheme in all $z$ range. This means that the performance degradation of the proposed scheme at the roof area mainly comes from the error in $z$ direction. It may be because the PSO tuning for $z$ direction is difficult when the height of all anchor nodes is the same, and further adjustments will improve the performance. To improve the roof area performance, the simplest solution is that the height of anchor nodes is increased to more than the upper bound of search area of $z$ (for example, $+1 \mathrm{~m}$ ).

Next, the scale down parameter in (18) is changed to $\hat{z}=\alpha g_{z}$ or fixed, and the three-dimensional RMSE is calculated. Here, $\alpha=1$ is our conventional scheme of [16]. The results in Fig. 8 showed that the performance with the fixed initial value $\hat{z}=1$ was degraded, and that the performances at lower height with the scaled-down initial value including $\hat{z}=0(\alpha=0)$ were improved. Then, in the same manner as Fig. 6 , the RMSE variance with small $\alpha$ in the same simulation is calculated. The result in Figs. 9 (a) and (b) shows that the variance is slightly smaller with a large $\alpha$ and a stable estimation regardless of the target node position will be conducted with larger $\alpha$. Hence, in the proposed scheme, $\alpha=0.3$ which has good RMSE performance in Fig. 8 and lower variance in Fig. 9 is adopted.

Consequently, it was shown that the proposed scheme could accurately estimate the location with less calculation complexity in three dimensional LS localization even when the height of the target and anchor node are close.

\section{Conclusion}

In this study we proposed the hybrid scheme of PSO which effectively searched in wide areas and NR which could conduct fine search for three-dimensional LS-based localization. Because the NR scheme sometimes terminates at local optimal solution, the high-complexity accurate grid is needed in the conventional scheme, while the proposed scheme achieves less-complexity and accurate estimation by adopting PSO coarse search. By numerical results it was shown that the least RMSE of estimation could be obtained with $1 / 3$ times calculation complexity compared to the conventional hybrid scheme of grid and NR. Also, except the upper height near anchor nodes, the proposed scheme has better RMSE performances in the $z$ direction.

\section{Acknowledgments}

This work is partially supported by the Telecommunications Advancement Foundation (TAF). The authors wish to thank for their support.

\section{References}

[1] I. Guvenc and C.C. Chong, "A survey on TOA based wireless localization and NLOS mitigation techniques," Communications Surveys \& Tutorials, IEEE, vol. 11, no. 3, pp. 107-110, Third Quarter 2009.

[2] J.J. Caffery, "A new approach to the geometry of TOA location," Proc. IEEE Vehicular Technology Conf., vol. 4, pp. 1943-1949, 2000.

[3] H.C. So, "Source localization: Algorithms and analysis," Handbook of Position Location: Theory, Practice and Advances, S.A. Zekavat and R.M. Buehrer, Eds., chapter 2, pp. 25-66. WileyIEEE Press, 2011.

[4] K. Fukuda and E. Okamoto, "Performance improvement of IMR-based NLOS detection in indoor ultra wide-band TOA localization," IEICE Trans. Fundamentals, vol. E95-A, no. 10, pp. 1658-1666, October 2012.

[5] J. Schroeder, S. Galler, K. Kyamakya, and T. Kaiser, "Three-dimensional indoor localization in Non Line of Sight UWB channels," Proc. IEEE International Conference on Ultra-Wideband (ICUWB2007), pp. 89-93, September 2007.

[6] J. Kennedy and R. Eberhart, "Particle swarm optimization," Proc. IEEE International Conference on Neural Networks, vol. 4, pp. 1942-1948, November 1995. 
[7] J.A. Parejo, A. Ruiz-Cortes, S. Lozano, and P. Fernandez, "Metaheuristic optimization frameworks: A survey and benchmarking," Soft Comput., vol. 16, no. 3, pp. 527-561, March 2012.

[8] J.H. Holland, "Adaptation in natural and artificial systems: An introductory analysis with applications to biology, control, and artificial intelligence," U Michigan Press, 1975.

[9] S. Kirkpatrick, C.D. Gelatt Jr., and M.P. Vecchi, "Optimization by simulated annealing," Science, vol. 220, no. 4598, pp. 671-680, May 1983.

[10] F. Glover, "Tabu search: A tutorial," Interfaces, vol. 20, no. 4, pp. 74-94, July 1990.

[11] P.-J. Chuang and C.-P. Wu, "An effective PSO-based node localization scheme for wireless sensor networks," Proc. Int'l Conf. Parallel and Distributed Computing, Applications and Technologies, pp.187-194, December 2008.

[12] G.S. Tewolde and J. Kwon, "Efficient WiFi-Based indoor localization using particle swarm optimization," Proc. Int'l Conf. Advances in Swarm Intelligence, pp. 203-211, 2011.

[13] T.N. Le, J.-W. Kim, and Y. Shin, "TDoA localization based on particle swarm optimization in UWB systems," IEICE Transactions on Communications, vol. E94B, no. 7, pp. 2013-2021, July 2011.

[14] S. Monica and G. Ferrari, "Impact of the number of beacons in PSO-based Auto-localization in UWB networks," Proc. International Conference on the Applications of Evolutionary Computation (EvoApplications13), pp. 42-51, April 2013.

[15] M.M. Noel, P.P. Joshi, and T.C. Jannett, "Improved maximum likelihood estimation of target position in wireless sensor networks using particle swarm optimization," Proc. Information Technology: New Generations, pp. 274-279, April 2006.

[16] E. Okamoto, M. Horiba, K. Nakashima, T. Shinohara, and K. Matsumura, "Particle swarm optimization-based low-complexity three-dimensional UWB localization scheme," Proc. International Conference on Ubiquitous and Future Networks (ICUFN2014), pp. 120-124, July 2014.

[17] B. Alavi and K. Pahlavan, "Modeling of the TOA-based distance measurement error using UWB indoor radio measurements," IEEE communications letters, vol. 10, no. 4, pp. 275-277, April 2006.

[18] S.J. Howard and K. Pahlavan, "Measurement and analysis of the indoor radio channel in the frequency domain," IEEE Trans. Instrumentation and Measurement, vol. 39, no. 5, pp. 751-755, October 1990.

[19] G. Bellusci, G.J.M. Janssen, J. Yan, and C.C.J.M. Tiberius, "Model of the distance and bandwidth dependency of TOA-based UWB ranging error," IEEE ICUWB 2008, 10-12 September 2008 . 